\title{
Theoretical Analysis of the Spatial Variability in Tillage Forces
}

\section{for Fatigue Analysis of Tillage Machines}

A. Abo Al-kheer ${ }^{1 *}$; M. Eid ${ }^{2}$; Y. Aoues ${ }^{2}$; A. El-Hami ${ }^{2}$; M. G. Kharmanda ${ }^{3}$; A. M. Mouazen ${ }^{4}$

1- Agricultural Engineering Department, Aleppo University, Syria

2- Rouen Mechanics Laboratory, National Institute of Applied Sciences, France

3- Faculty of Mechanical Engineering, Aleppo University, Syria

4- Environmental Science and Technology Department, Cranfield University, MK43 0AL, United Kingdom

* Corresponding author: Tel: 0096323211410, E-mail: a.aboalkheer@hotmail.com

12

13 Abstract

14 This paper presents a new theoretical model to describe the spatial variability in tillage forces for

15 the purpose of fatigue analysis of tillage machines. The proposed model took into account both

16 the variability in tillage system parameters (soil engineering properties, tool design parameters

17 and operational conditions) and the cyclic effects of mechanical behaviour of the soil during

18 failure ahead of tillage tools on the spatial variability in tillage forces. The stress-based fatigue

19 life approach was used to determine the life time of tillage machines, based on the fact that the

20 applied stress on tillage machines is primarily within the elastic range of the material. Stress

21 cycles with their mean values and amplitudes were determined by the rainflow algorithm. The

22 damage friction caused by each cycle of stress was computed according to the Soderberg criterion

23 and the total damage was calculated by the Miner's law. 
24 The proposed model was applied to determine the spatial variability in tillage forces on the shank 25 of a chisel plough. The equivalent stress history resulted from these forces were calculated by 26 means of a finite element model and the Von misses criterion. The histograms of mean stress and

27 stress amplitude obtained by the rainflow algorithm showed significant dispersions. Although the 28 equivalent stress is smaller than the yield stress of the material, the failure by fatigue will occur 29 after a certain travel distance. The expected distance to failure was found to be $=0.825 \times$ $3010 \quad$. It is concluded that the spatial variability in tillage forces has significant effect on the 31 life time of tillage machines and should be considered in the design analysis of tillage machines

32 to predict the life time. Further investigations are required to correlate the results achieved by the 33 proposed model with field tests and to validate the proposed assumptions to model the spatial 34 variability in tillage forces.

35 Keywords: spatial variability; tillage forces; tillage machines; fatigue analysis; life time.

\section{1- Introduction}

38 Mechanical loads on tillage machines show considerable variability due to the variability in 39 tillage system parameters and the mechanical behaviour of soil during failure. The variability in 40 tillage system parameters reflects the variability in soil engineering properties and the variations 41 in tool design parameters and operational conditions. The variability in soil engineering 42 properties is resulted from the heterogeneity of agricultural soils. The variations in tool design 43 parameters are due to the manufacturing processes, while the variations in operational conditions 44 are due to the fact that these parameters are not completely controlled during tillage operation.

45 The crescent manner of soil failure for wide and narrow tines with depth/width ratio less than 5 46 tend to push the soil upward and forward [1]. This failure pattern involves the development of 47 successive shear planes in front and at the side of tillage tools, which leads to distinct soil failure 
48 blocks (crescents) as the tine moves forward through the soil. The repeated formation of soil 49 crescents creates cyclic loading on the tillage tool. Stafford [2] identified two types of soil failure, 50 namely the brittle failure and the flow failure. Rajaram and Erbach [3] reported the following four 51 soil failure patterns: 1) collapse failure, 2) fracture failure, 3) chip-forming failure and 4) plastic 52 and frictional flow failures. However, they attributed the changes of soil failure patterns to two 53 main factors: the soil type and the moisture content. In other work, Rajaram and Erbach [4] 54 reported that the variations in soil failure patterns are mostly affected by the soil moisture content, 55 soil type, tillage implement type and tillage speed. Factors controlling the soil mechanical 56 behaviour during failure under load are not completely understood/known. In addition, there are 57 no available models that can estimate the effects of soil failure on tillage forces.

58 Several analytical (e.g. McKyes and Ali [5]; Grisso et al. [6]) and numerical models (e.g. Shen 59 and Kushwaha [7]; Mouazen and Neményi [8]) of soil-tool interaction have been developed to 60 predict tillage forces for assigned tillage system parameters. Furthermore, the effects of tillage 61 system parameters on tillage forces have been investigated [1,9]. Although, these models have 62 shown good agreements with experimental observations for specific test conditions, there is still 63 no well-defined, generalised theoretical model to predict the behaviour of soil-tool interactions 64 [10]. In order to take into consideration the fact that tillage system parameters are variable during 65 tillage operations, Abo Al-kheer et al. [11] proposed a methodology for modeling the variability 66 in tillage forces derived from the variability in tillage system parameters. In another report, they 67 integrated this variability into the reliability analysis for achieving reliable tillage machines [12].

68 The variability in tillage forces due to the soil failure has been observed in many works in the 69 literature. James and Shrini [13] reported that the large variations in the horizontal force on a 70 plough can be attributed to two factors. These are related to the soil failure and to the within-field 71 spatial variability in soil properties. They confirmed that both of these factors lead to relatively 72 large changes in the horizontal force within a short time period. Makanga et al. [14] studied the 
73 effects of the tine rake angle and the aspect ratio in a laboratory glass-sided soil bin with a dry 74 compacted loam soil with $5.2 \%$ (d.b.) moisture content. They concluded that the horizontal and 75 vertical soil reactions under dry soil conditions were cyclic in nature and in phase. The cyclic

76 variations in the soil reactions were due to the soil failure patterns being repetitive and cyclic in 77 nature throughout the tine travel. Due to variability in soil properties and operational conditions 78 the frequency and amplitude of these cycles vary significantly.

79 Form a fatigue analysis viewpoint, it is essential to account for the effects of the variability in 80 tillage forces on the resulted stress on tillage machines. The current state of knowledge suggests 81 that there are only experimental works available to estimate the spatial variability in tillage forces $82[15,16,17]$. These methods do not provide a tool for estimating the life time of tillage tools due to 83 fatigue as it cannot account for all affecting parameters.

84 The main objective of this work is to propose a model for estimating the variability in tillage 85 forces for the purpose of fatigue analysis of tillage machines. The proposed model is applied to 86 estimate the spatial variability in tillage forces on the shank of a chisel plough and the expected 87 distance to failure is calculated. This model is presented in the first part of this work. The second 88 part presents the stress-based fatigue life approach used to estimate the life time of tillage 89 machines.

90

\section{$91 \quad$ 2- Materials and methods}

\section{$92 \quad 2-1$ Modeling the spatial variability of tillage forces}

\section{$93 \quad$ 2-1-1 Basic assumptions for the proposed model}

94 The basic assumptions behind the proposed model are that 1) the spatial variability of tillage 95 forces derived from the variability in tillage system parameters is random, reflecting the 96 heterogeneity in agricultural soils, the uncontrolled field operational conditions during tillage and 
97 the manufacturing tolerances of tool design parameters and 2) the spatial variability of tillage

98 forces derived from the mechanical behavior of soil failure is cyclic, reflecting the repeated

99 formation of soil crescents in front of the tool. In addition, we suppose that the total tillage force

100 is the sum of two types of forces, namely the global tillage force and the local tillage force. The

101 global tillage force is due to the tillage system parameters (soil engineering properties, tool design

102 parameters and operational conditions) and the local tillage force is due to the soil failure of

103 cyclic nature.

104 Conventionally, a tillage force is determined by its horizontal and vertical components.

105 Therefore, the horizontal and vertical forces can be calculated, according the earlier assumption,

106 by Equations (1) and (2).

$$
\begin{array}{ll}
= & + \\
= & +
\end{array}
$$

107 where is the global horizontal force in $\mathrm{kN}$, is the local horizontal force in $\mathrm{kN}$, is the 108 global vertical force in $\mathrm{kN}$ and is the local vertical force in $\mathrm{kN}$.

109 The variability in the global tillage forces ( , ) can be modelled using the methodology 110 proposed by Abo Al-kheer et al. [11]. This methodology is based on the estimation of tillage 111 forces according to the McKyes-Ali model accounting for the variability in tillage system 112 parameters. Abo Al-kheer et al. [11] subdivided the tillage system parameters contributing to the 113 global tillage forces into three main categories: 1) soil engineering properties including soil bulk 114 density, soil cohesion, internal friction angle, soil-metal friction angle and soil-tool adhesion), 2) 115 tool design parameters including tool width and rake angle and 3) operational conditions 116 including tool working depth, surcharge pressure and tool speed. A combination of graphical and 117 quantitative techniques was proposed for modeling the variability in soil engineering properties 118 and two statistical tests were used to select the probability distributions of these properties, 
namely the chi-square test and the Kolmogorov-Smirnov test. A total of 57 variations of soil

120 engineering properties, representing 57 different soil samples were considered for implementing

121 our mixed technique approach [11]. The probabilistic characteristics of these properties are given

122 in Table 1

123 The local tillage forces ( , ) have been observed in many works in the literature but there

124 are no available models can be used to estimate these forces. However, the majority of reports are

125 attributing these forces to nearly the same parameters contributing to the global tillage forces [4].

126 Therefore, we assume that the local tillage force components can be estimated as a percentage of

127 the global tillage force components as shown in Equations (3) and (4).

$$
=\text {. }
$$

$$
=\text {. }
$$

128 where is the percentage of the local tillage force to the global tillage force. According to the 129 assumptions in Equations 3 and 4 global and local forces are related by the tillage system 130 parameters (soil engineering properties, tool design parameters and operational conditions). The 131 high values of corresponding to a brittle soil failure and the little values of corresponding to a 132 flow soil failure. In other words, the values of the local tillage forces $(\quad, \quad$ ) are important for 133 the brittle soil failure since the force cyclic pattern is much more pronounced that with flow 134 failure, while the values of these forces are nearly zero when the soil failure is of flow type [10].

135 The linear correlation between the global and local tillage forces may not be accurate for all soil 136 texture types and all operational conditions. Thus, more work should be done to investigate the 137 relationship between the global and local tillage forces.

138 Based on the earlier assumptions, the spatial variability in tillage forces can be represented by the 139 spatial variability in the global and local tillage forces, as shown in Equations (5) and (6).

$$
\begin{aligned}
& ()=()+() \\
& ()=()+()
\end{aligned}
$$


140 where represents the spatial variability in the global horizontal force in $\mathrm{kN}$, represents

141 the spatial variability in the local horizontal force in $\mathrm{kN}$, represents the spatial variability in

142 the global vertical force in $\mathrm{kN}$, represents the spatial variability in the local vertical force in

$143 \mathrm{kN}$ and is the distance travelled in .

\section{$144 \quad 2-1-2$ Modeling the spatial variability in the global tillage forces}

145 The spatial variability in the global tillage forces $(, \quad$ ) is resulted from the spatial variability

146 of soil resistance and uncontrolled operational conditions. This spatial variability can be

147 attributed to several factors, e.g., the characteristics of the field, the geography and topography of

148 the field and the soil management system (no-till, reduced till or conventional tillage). Therefore,

149 the spatial variability in the global tillage forces changes from one location to another within the

150 same field and from field to field.

151 To take the earlier observations into account, we modelled the spatial variability in the global

152 tillage forces with the following assumptions: 1) the spatial variability in the global tillage forces

153 is linear and 2) the distance between two successive changes of the values of global tillage

154 forces is random. The linearity of the spatial variability in the global tillage forces between the

155 global tillage force samples may not be an accurate assumption. However, the increase of the

156 global tillage force samples improves the accuracy of this model. Taking the distance as a

157 random variable allows considering the variability in the field characteristics over the distance

158 Based on these assumptions, the spatial variability in the global tillage forces can be expressed as

159 in Equations (7) and (8). An illustration of the spatial variability in the global tillage forces over 160 the distance is shown in Figure 1.
()$=()+(+1)-$
( ) $\frac{-\sum \text { ( ) }}{()}$
$=1, \ldots$,
()$=()+(+1)-$
( ) $\frac{-\sum()}{()}$
$=1, \ldots$, 
161 where ( ) is the $\mathrm{i}$ th global horizontal force sample in $\mathrm{kN}, \quad$ ( ) is the $\mathrm{i}$ th global vertical

162 force sample in $\mathrm{kN}$, is the distance between two successive changes of the global tillage force

163 values in,$\sum \quad$ ( ) is the cumulative sum of ( ) for $=1$ to -1 and is the number of

164 the global tillage force samples.

\section{2-1-3 Modeling the spatial variability in the local tillage forces}

166 As mentioned before, the soil failure creates cyclic loading on tillage tools by the repeated

167 formation of soil crescents. The global tillage forces are calculated at failure when the tillage

168 forces achieve their maximum values. The local tillage forces reach their maximum values at

169 failure and then drop down after the first soil block has formed and these forces will increase to

170 form the second soil block until achieve failure and so on. Therefore, we can imagine that the

171 total tillage forces fluctuate below the global tillage forces.

172 Based on the fact that the effect of the soil failure in the tillage forces is cyclic, the sinusoid 173 function was used to describe the spatial variability in the local tillage forces with the 174 amplitude and cycle length . Therefore, the spatial variability of tillage forces can be 175 expressed as in Equations (9) and (10) and illustrated as in Figure 2. The terms - ( ) and

176 - ( ) are added to these Equations to keep the values of total tillage forces fluctuate below the 177 values of global tillage forces.

$$
\begin{aligned}
& ()=() \sin 2 \frac{-\sum()-\sum()}{()}-()=1, \ldots, \\
& ()=() \sin 2 \frac{-\sum()-\sum()}{()}-()=1, \ldots,
\end{aligned}
$$

178 where ( ) is the $\mathrm{j}$ th local horizontal force in $\mathrm{kN}, \quad(\mathrm{)}$ is the $\mathrm{j}$ th local vertical force in $\mathrm{kN}$, 179 ( ) is the cycle length of the jth cycle in , is the number of calculated values in a cycle and 180 is the number of cycles between two successive changes of the global tillage forces. 
182 By combining the spatial variability in the global and local tillage forces and taking into account 183 the assumption that the local tillage force components can be estimated as a percentage of the 184 global tillage force components, it concluded that the spatial variability in tillage forces can be 185 represented by the following five parameters:

$$
\begin{aligned}
& ()=\left(P_{H G}, S_{1}, S_{2},\right) \\
& ()=\left(P_{V G}, S_{1}, S_{2},\right)
\end{aligned}
$$

186 All of these parameters can be considered as variables to represent the variability in the forces on

187 the tillage tool during the tillage operation, as shown in Figure 3.

\section{2-1-5 Special cases}

189 Two special cases of spatial variability in tillage forces, namely, at constant global tillage forces 190 and at insignificant local tillage forces are shown in Figures 4 and 5, respectively. The first case 191 supposes that all tillage system parameters do not vary during tillage. This assumption may be 192 suitable for quasi-homogeneous soils and when the variations in the operational conditions are 193 not important. The second case can be used to represent the spatial variability in tillage forces 194 when the soil failure is of flow type. However, in most cases, both the global and local tillage 195 forces should be taken into consideration in the estimation of the spatial variability in tillage 196 forces.

\section{2-2 Stress-based fatigue life}

199 The stress-based fatigue life approach is generally characterized by a high-cycle fatigue 200 methodology, and is widely used in design applications where the applied stress is primarily 201 within the elastic range of the material and the resulting fatigue life is long. The basis of the 
stress-based fatigue life approach is the stress (S)-number of cycles to failure $(\mathrm{N})$ curve, also

203 known as a Wöhler curve. The S-N curve is a graph of the amplitude of a cyclical stress against 204 the logarithmic scale of cycles to failure. In some materials, particularly ferrous alloys, the S-N curve flattens out eventually, so that below a certain limit, called the fatigue limit or the 206 endurance limit (typically > 10 cycles), the material may not fail and can be cycled infinitely

207 [18] (curve a in Figure 6). For some other materials such as aluminum and copper alloys, no 208 fatigue limit exists. In such cases, the fatigue strength for a given number of cycles (e.g. $1 \times 10$ 209 cycles) must be specified [19] (curve b in Figure 6).

210 The S-N curve for a material, that has a fatigue limit such as steel, can be expressed as in 211 Equation (13).

$$
=\cdot \quad<
$$

212 where is the stress amplitude in MPa, is the regression intercept (also called the fatigue 213 strength coefficient) in MPa, is the regression slope (also called the fatigue strength exponent), 214 is the number of cycles and is the number of cycles corresponding to the fatigue limit .

215 The most basic S-N curves are generated using a fully-reversed stress, where the ratio (R) 216 between the maximum and minimum stress is equal to -1 . When the stress applied on a structure 217 is constant over the structure life and the ratio (R) is equal to -1, the Equation (13) can be used 218 directly to determine the number of cycles to failure i.e. the fatigue life. If the number of cycles to 219 failure is greater than the number of cycles corresponding to the fatigue limit , it can be 220 accepted that the structure has an infinite life.

221 When the ratio $\mathrm{R}$ is not equal to -1 , a Haigh diagram is usually used to estimate the fatigue life.

222 This diagram plots the mean stress along the $\mathrm{x}$-axis and the stress amplitude along the $\mathrm{y}$-axis and 223 the lines of constant life are drawn through the data points. A very substantial amount of testing is 224 required to generate a Haigh diagram, and it is usually impractical to develop curves for all 
225 combinations of mean and amplitude stresses. Therefore, several empirical criteria that relate the 226 stress amplitude to the mean stress have been developed to address this difficulty. These criteria 227 define various curves to connect the fatigue limit on the stress amplitude axis to either the yield 228 strength or the ultimate strength on the mean stress axis [20]. The zone under the curves defined 229 the safe zone against fatigue while the zone above the curves represents the failure zone. Figure 7 230 illustrates three of these criteria, namely the criterions of Goodman, Gerber and Soderberg.

231 Kwofie [21] proposed a function, presented in Equation (14), to take into account the effect of 232 mean stress. This function allows determining the stress amplitude according to the material 233 constant, material properties, number of cycles to failure and to different fatigue criteria.

$$
=1--
$$

234 where is a numerical constant, representing the mean stress sensitivity of the material, is the 235 mean stress in MPa and is the ultimate strength in MPa. The value of the numerical constant 236 depends on the fatigue criterion (Goodman, Gerber, Soderberg, etc.).

237 In practice, a structure (e.g. tillage machines) is exposed to a random stress. In such cases, the 238 random stress should be reduced to a series of simple cyclic stresses using counting methods, e.g., 239 range pair method and rainflow method [22]. The rainflow method is the most popular and widely 240 used in practice [23]. It allows one to determine the amplitude and mean value $(\quad, \quad)$ for each 241 stress cycle at a fixed time interval or a distance interval . The damage friction caused by the 242 ith cycle of stress can be computed by Equation (15).

$$
=\frac{1}{(, \quad)}
$$

243 where ( , ) is the number of cycles to failure according to the amplitude and the 244 mean value . In this work, the Soderberg criterion was used to determine , based on the 
245 fact that the applied stress is primarily within the elastic range of the material. According to this

246 criterion, $=\quad[21]$ and was calculated as in Equation (16).

$$
=\overline{1-}
$$

247 The total damage, caused by all cycles, can be computed by a cumulative damage model. More 248 than 60 fatigue damage models have been proposed for this purpose. However, the linear damage 249 rule (Miner's law) is still dominantly used because of its simplicity in addition to its sufficient 250 accuracy [24]. For these reasons the Miner's law was used in this work. The Miner's law assumes 251 that the total damage can be expressed as the sum of damage frictions, as shown in Equation (17).

252 Failure is assumed to occur when $\geq 1$.

253 where is the number of cycles determined by the rainflow algorithm for the time interval or 254 the distance interval . If the total damage is calculated for a distance interval , which is the 255 case of our work, the expected travel distance to failure can be calculated by dividing the distance 256 interval by the total damage, i.e. = , to fulfill the assumption that the failure will occur 257 when $\geq 1$.

\section{3- Numerical application}

260 3-1 Modeling the spatial variability in tillage forces

261 The proposed model, presented in Section 2.1, is implanted in MATLAB program (Mathworks

262 INC. 2008) to model the spatial variability in tillage forces on the shank of a chisel plough shown 263 in Figure 8. The shank cross-section is rectangular of height $h=58$ and width $=32$, 264 as shown in Figure 9. 
265 Abo Al-kheer et al. [11] found that the variability in the global horizontal and vertical forces

266 followed lognormal distributions. The distribution parameters of these forces were $\quad=0.872$,

$267=0.449, \quad=0.004$ and $=0.447$, where and are the scale and shape

268 parameters of a lognormal distribution, respectively. The correlation coefficient between and

269 was found to be $(, \quad)=0.93$. Therefore, the variability in the local horizontal and

270 vertical forces should have lognormal distributions with the following distribution

271 parameters $=\ln ()+, \quad=\quad, \quad=\ln ()+$ and $=$. In this

272 work, was selected to be equal 0.2 for brittle failure. Furthermore, we assumed

273 that and have normal distributions with the following distribution parameters =

$27410,=0.1, \quad=0.05$ and $=0.1$, where and are the location and scale

275 parameters of a normal distribution, respectively. The distribution parameters of the main model

276 parameters, as described in Equations (11) and (12), are listed in Table 2.

277 To generate correlated tillage forces and , two non-correlated normalized variables

278 and were generated by the MATLAB function "normrnd" and then the random values of

279 and were calculated using two transformations [25,26]. The first one transforms non-

280 correlated normalized variables and to correlated normalized variables and and the

281 second one transforms correlated normalized variables to correlated tillage forces and .

282 The spatial variability of the horizontal and vertical forces can then be shown in Figure 10 for a

283 distance of 1000 .

284 From Figure 10, it can be observed that a clear correlation exists in the spatial variability between

285 the horizontal and vertical forces. This is resulted from the correlation between the global

286 horizontal and vertical forces $((, \quad)=0.93)$. Another observation is the correlation

287 between the global tillage forces $(, \quad)$ and the local tillage forces $(, \quad)$. The increase

288 of global tillage forces increases the amplitudes of local tillage forces and vice-versa. These 
289 increases in force amplitudes are marked in cycles in Figure 10. This is caused by the calculation

290 of the local tillage forces as a percentage of the global tillage forces.

\section{$291 \quad 3-2$ Equivalent stress history}

292 The equivalent stress, resulted from the tillage forces, was calculated according to the Von misses 293 criterion. Firstly, the point of the maximum equivalent stress was determined by means of the 294 finite element (FE) method and ANSYS program (ANSYS INC. V11). Figure 11 shows the 295 meshed model, boundary conditions and the point of maximum equivalent stress (in MPa) 296 determined for the mean values of tillage forces $=2.641$ and $=1.106$. Then, the 297 equivalent stress, presented in Figure 12, was calculated at the point of maximum equivalent 298 stress using the finite element model, implemented in the CALFEM toolbox of MATLAB [27].

299 The rainflow algorithm [23] was used to extract the stress cycles with their amplitude and mean 300 values. The histograms of stress amplitude and mean stress are shown in Figure 13. Both the 301 histograms indicate that the dispersions of mean stress and stress amplitude are significant. This 302 reflects the high dispersions of the spatial variability in tillage forces.

\section{$303 \quad 3-3$ Expected distance to failure}

304 The material constants (the regression intercept and the regression slop) used to calculated the 305 expected distance to failure are $\mathrm{a}=754 \mathrm{MPa}$ and $=0.121$. The yield stress of the material 306 is $=250 \mathrm{MPa}$. The total damage was calculated over the distance $(=980.902)$ 307 according to Equation (17). The total damage is equal to $=1.189 \times 10$. By dividing the 308 distance by the total damage , the expected distance to failure is $=0.825 \times 10$. 309 Despite the fact that the equivalent stress is smaller than the yield stress (Figure 10), the failure 310 will occur after a certain distance . This example shows the significant effect of the spatial 311 variability in tillage forces on the life time of tillage machines. Since agricultural soils are 
312 characterized to be high spatial variability [17], it is expected that this variability will reduce the

313 life time of tillage tools.

314 The expected distance to failure is plotted as a function of the shank cross-section dimensions

$315(, h)$ in Figure 14. For all combinations of and $h$, the equivalent stress is smaller than the yield

316 stress. The minimum distance to failure $\quad=2.004 \times 10 \quad$ occurs with $=25$

317 and $h=55$, the maximum distance to failure $=9.213 \times 10 \quad$ occurs with

$318=35$ and $h=70$. It is noted that the augmentation of the shank cross-section

319 dimensions increases the distance to failure. This is due to the fact that the augmentation of the

320 shank cross-section dimensions augments the resistance to failure by fatigue and by consequence

321 augments the distance to failure. The Figure 14 allows one determining the shank dimensions

322 according to the required distance to failure, e.g., for $=10 \times 10$ the shank dimensions

323 are $=25$ and $h=65$.

324 To investigate the effect of the percentage of the local tillage forces to the global tillage forces ( )

325 on the expected distance to failure ( ), the percentage is plotted against the logarithmic scale

326 of in Figure 15. It is observed that with an increase of from 01 to 0.4 , a reduction of

327 of $1.2 \times 10 \quad$ will take place, meaning that the reduction of due to the augmentation of is

328 very significant. Indeed, the increase of the value of the percentage augments the amplitudes of

329 the fatigue cycles and as a result reduces the distance to failure (Equations 15, 16 and 17).

330 Therefore, to reduce the values, (by consequence, the values of the local tillage forces) it is

331 recommended to perform the tillage operation when the moisture content is closed to the liquid

332 limits, where the soil conditions became most favorable for soil-working. This can improve

333 significantly the expected distance to failure and by consequence the life time of tillage machines. 
337 A new model to describe the spatial variability in tillage forces and the methods used to calculate

338 the life time of tillage machines are presented in this paper. The proposed model was applied to 339 determine the spatial variability in tillage forces for the shank of a chisel plough and the expected 340 distance to failure was caculated. The expected distance to failure for the shank cross section 341 of $h=58$ and $=32$ was found to be $=0.825 \times 10$. In addition, different values 342 of $h$ and could be used to calculate the expected distance to failure. Based on this work the 343 following conclusions can be drawn:

344 - The spatial variability in tillage forces can be modelled by only five random parametes $345 \quad\left(\mathrm{P}_{\mathrm{HG}}, \mathrm{P}_{\mathrm{VG}}, \mathrm{S}_{1}, \mathrm{~S}_{2}, \tau\right)$

346 - The spatial variability in tillage forces has singificant effect on the equivalent stress history.

347 - The dispersions of mean stress and stress amplitude of the stress cycles are important.

348 - The failure by fatigue will occur even the equilvalent stress is smaller than the yield stress of 349 the material.

350 - The shank cross-section dimensions have a significant impact on the distance to failure $(=2.004 \times 10 \quad, \quad=9.213 \times 10 \quad$ ).

352 - The effect of the percentage of the local tillage forces to the global tillage forces ( ) on the 353 expected distance to failure ( ) is very significant.

354 A further research is needed to investigate the relathionships between the global and local tillage 355 forces $\left(\mathrm{P}_{\mathrm{HG}}, \mathrm{P}_{\mathrm{VG}}\right)$ and the variability in the others parameters $\left(\mathrm{S}_{1}, \mathrm{~S}_{2}, \tau\right)$. In addition, a further 356 study is needed to validate the results achieved in this paper with experiment. 
362 [1] R.J. Godwin, A review of the effect of implement geometry on soil failure and implement 363 force, Soil \& Tillage Research 97( 2007) 331-340.

364 [2] J.V. Stafford, Force prediction models for brittle and flow failure of soil by draught tillage 365 tool, Journal of Agricultural Engineering Research 29(1984) 51-60.

366 [3] G. Rajaram, D.C. Erbach, Soil failure by shear versus modification by tillage: A review, 367 Journal of Terramechanics 33(1996) 265-272.

368 [4] G. Rajaram, D.C. Erbach, Drying stress effect on mechanical behaviour of a clay-loam soil, 369 Soil \& Tillage Research 49(1998) 147-158.

370 [5] E. McKyes, O.S. Ali, The cutting of soil by narrow blades, Journal of Terramechanics 371 14(1977) 43-58.

372 [6] R.D. Grisso, J.V. Perumpral, C.S. Desai,.A soil-tool interaction model for narrow tillage tools, 373 No. 80-1518 ASAE, St Joseph, MI, 1980.

374 [7] J. Shen, R.L. Kushwaha, Quick finite element analysis of soil tool interactions, Computers 375 and Electronics in Agriculture 9(1993) 289-299.

376 [8] A.M. Mouazen, M. Neményi, Finite element analysis of subsoiler cutting in non377 homogeneous sandy loam soil, Soil \& Tillage Research 51(1999) 1-15.

378 [9] R.J. Godwin, M.J. O'Dogherty, C. Saunders, A.T. Balafoutis, A force prediction model for 379 mouldboard ploughs incorporating the effects of soil characteristic properties, plough geometric 380 factors and ploughing speed, Biosystems Engineering 97(2007) 117-129.

381 [10] H.P.W. Jayasuriya, V.M. Salokhe, A review of soil-tine models for a range of soil, Journal 382 of Agricultural Engineering Research 79(2001) 1-13. 
383 [11] A. Abo Al-kheer, M.G. Kharmanda, A. El-hami, Probabilistic analysis of soil tillage forces, 384 Proceedings of the 18th ISTRO Conference, August 2009, Izmir, Turkey.

385 [12] A. Abo Al-kheer, A. El Hami, M.G. Kharmanda, A.M. Mouazen, Reliability-Based Design 386 for Soil Tillage Machines, Journal of Terramechanics 48(2011) 57-64.

387 [13] L.G. James, K.U. Shrini, An improved technique for agricultural implement draught 388 analysis, Soil \& Tillage Research 35(1995) 175-182.

389 [14] J.T. Makanga, V.M. Salokhe, D. Gee-Clough, Effects of tine rake angle and aspect ratio on 390 soil reactions in dry loam soil, Journal of Terramechanics 34(1997) 235-250.

391 [15] D. Sirjacobs, B. Hanquet, F. Lebeau, M.F. Destain, On-line soil mechanical resistance 392 mapping and correlation with soil physical properties for precision agriculture, Soil and Tillage 393 Research 64(2002) 231-242.

394 [16] B. Hanquet, D. Sirjacobs, M.F. Destain, Analysis of soil variability measured with a soil 395 strength sensor, Precision Agriculture 5(2004) 227-246.

396 [17] A.M. Mouazen, H. Ramon, Development of on-line measurement system of bulk density 397 based on on-line measured draught, depth and soil moisture content, Soil and Tillage Research $398 \quad 86(2006) 218-229$.

399 [18] J. Schijve, Fatigue of structures and materials in the 20th century and the state of the art, 400 International Journal of Fatigue 25(2003) 679-702.

401 [19] I. Marines, X. Bin, C. Bathias, An understanding of very high cycle fatigue of metals, 402 International Journal of Fatigue 25(2003) 1101-1107.

403 [20] G.P. Sendeckyj, Constant life diagrams - a historical review, International Journal of Fatigue $404 \quad 23(2001) 347-353$. 
405 [21] S. Kwofie, An exponential stress function for predicting fatigue strength and life due to 406 mean stresses, International Journal of Fatigue 23(2001) 829-836.

407 [22] J.A. Bannantine, J.J. Comer, J.L. Handrock, Fundamentals of metal fatigue analysis, Prentice 408 Hall, New Jersey, 1990.

409 [23] A. Nieslony, Determination off ragments of multiaxial service loading strongly influencing 410 the fatigue of machine components, Mechanical Systems and Signal Processing 23(2009) 27124112721.

412 [24] A. Fatemi, L. Yang, Cumulative fatigue damage and life prediction theories: a survey of the 413 state of the art for homogeneous materials, International Journal of Fatigue 20(1998) 9-34.

414 [25] A. Der Kiureghian, P-L. Liu, Structural reliability under incomplete probability information, 415 Journal of Engineering Mechanics 112(1986) 85-104.

416 [26] A. Haldar, S. Mahadevan, Probability, reliability, and statistical methods in engineering 417 design, John Wiley \& Sons, New York, 2000.

418 [27] P.E. Austrell, O. Dahlblom, J. Lindemann, A. Olsson, K.G. Olsson, K. Persson, H. 419 Petersson, M. Ristinmaa, G. Sandberg, P.A. Wernberg, CALFEM; A finite element toolbox to 420 MATLAB, Version 3.4, Lund : Structural Mechanics, LTH, Sweden, 2004.

421 
Tables

433 Table 1: Probabilistic characteristic of soil engineering properties (Abo Al-kheer et al. [11])

\begin{tabular}{|c|c|c|}
\hline Soil engineering properties & Type of distribution & Distribution parameters \\
\hline Soil density, kN.m & Lognormal & $=0.13,=2.7$ \\
\hline Soil cohesion, $\mathrm{kPa}$ & Weibull (2P) & $=15.51,=1.66$ \\
\hline Internal friction angle, deg & Normal & $=32,=3.96$ \\
\hline Soil-tool friction angle, deg & Weibull (3P) & $=-64.08, \quad=87.14, \quad=31.52$ \\
\hline Soil-tool adhesion, $\mathrm{kPa}$ & Exponential & $=0.76$ \\
\hline
\end{tabular}


450 Table 2: Distribution parameters of the model's parameters

\begin{tabular}{|c|c|c|c|}
\hline Model's parameters & Distribution type & \multicolumn{2}{|c|}{ Distribution parameters } \\
\hline$[\quad]$ & Lognormal & $=0.872$, & $=0.449$ \\
\hline$[\quad]$ & Lognormal & $=0.004$, & $=0.447$ \\
\hline [ ] & Normal & $=10$ & \\
\hline \multirow[t]{2}{*}[\begin{array}{ll}{}&{]}\end{array}]{} & Normal & $=50$ & \\
\hline & Deterministic & $=0.2$ & \\
\hline
\end{tabular}

451

452

453

454

455

456

457

458

459

460

461

462

463

464

465

466

467

468

469

470 


\section{$472 \quad$ Figures}

473

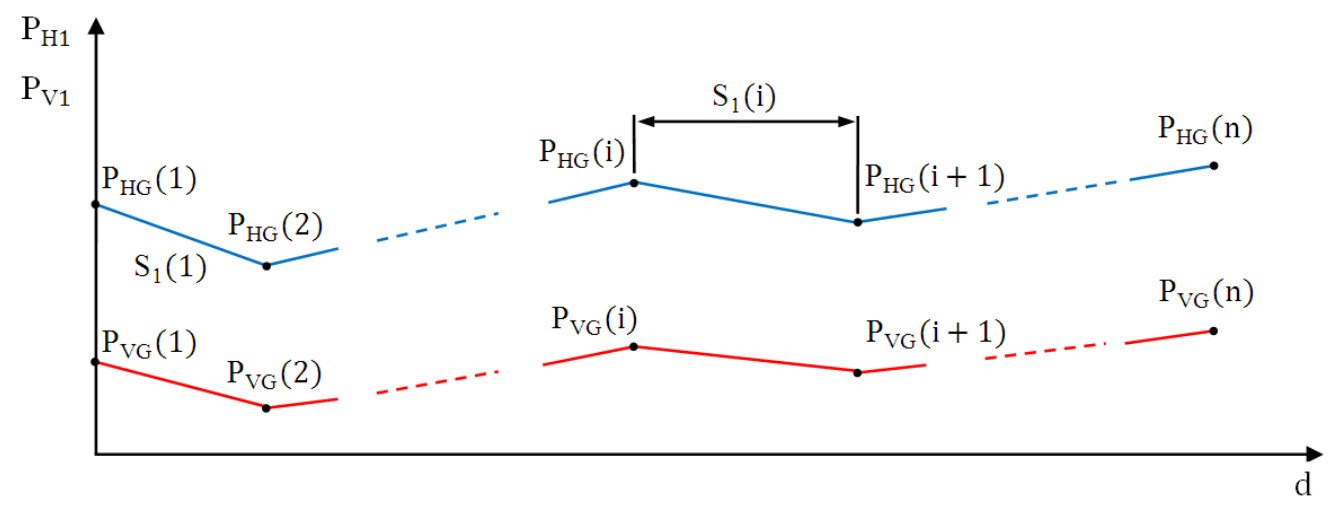

475

Figure 1: Illustration of the spatial variability in the global tillage forces

476

477

478

479

480

481

482

483

484

485

486 


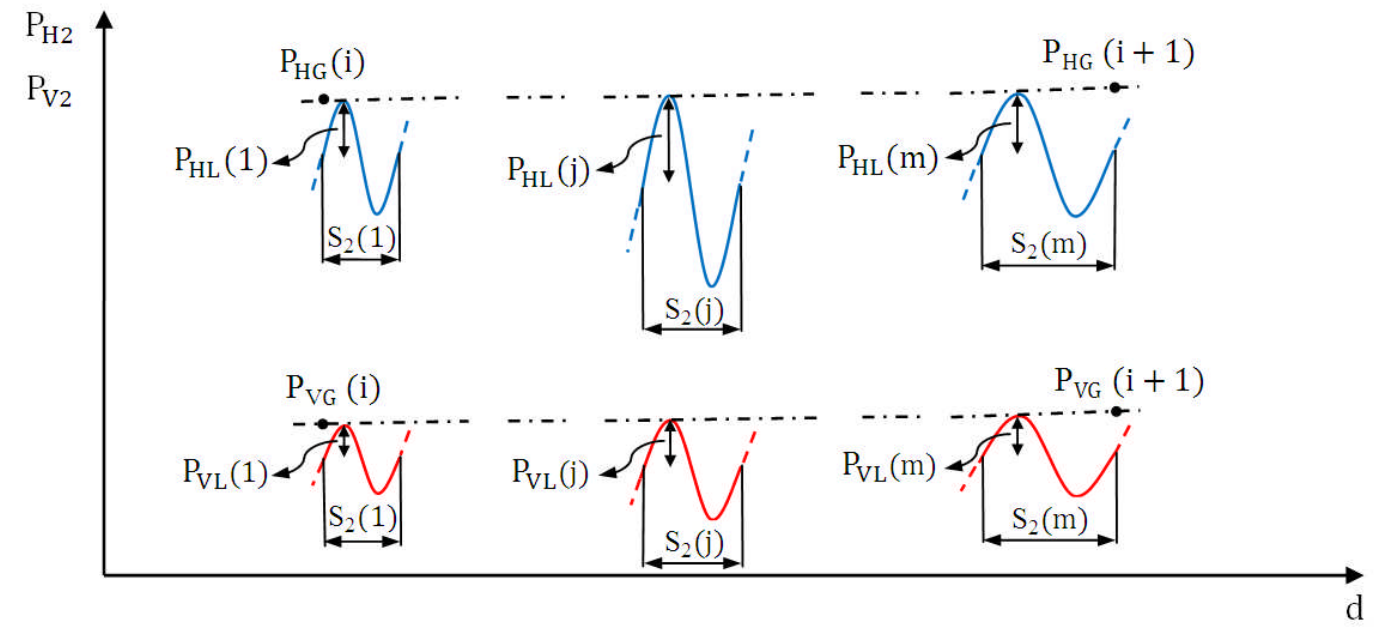

Figure 2: Illustration of the spatial variability in the local tillage forces

490

491

492

493

494

495

496

497

498

499

500

501 


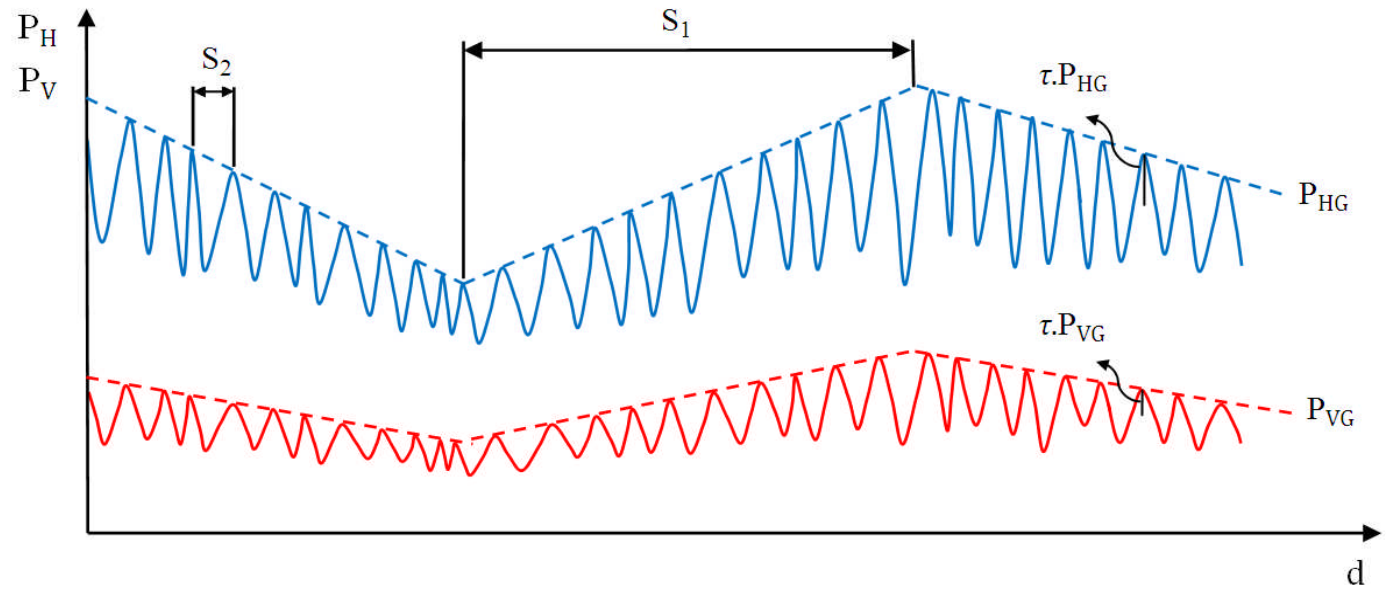




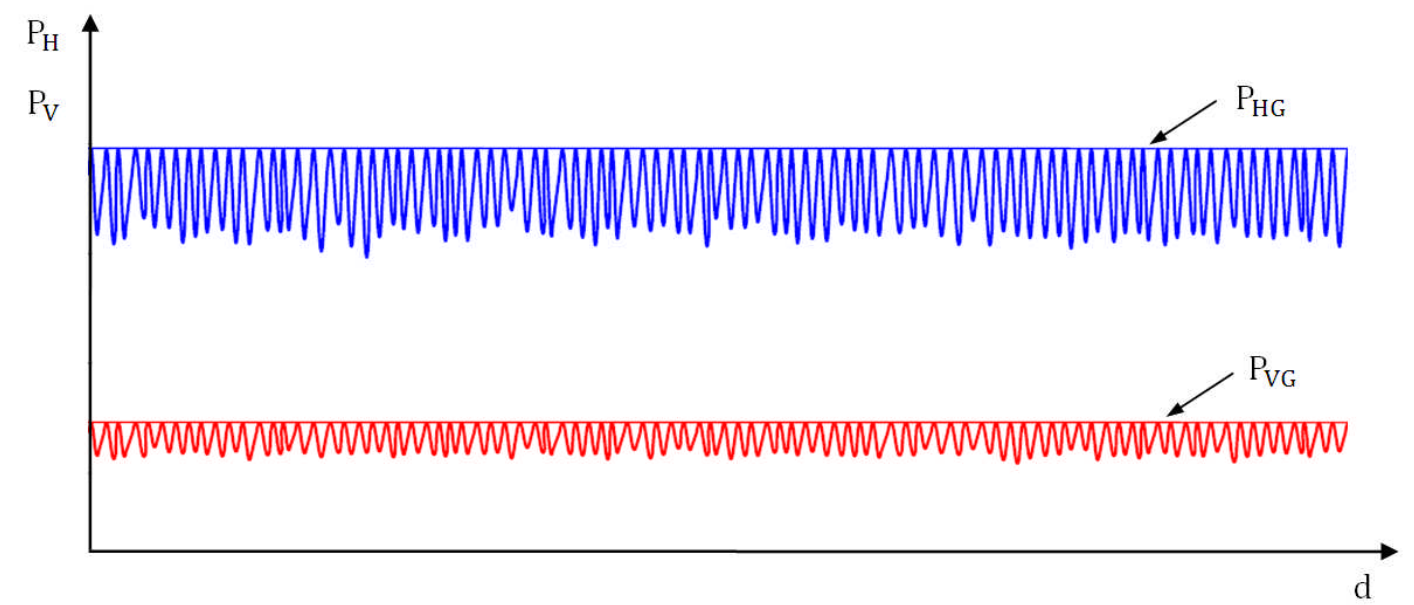




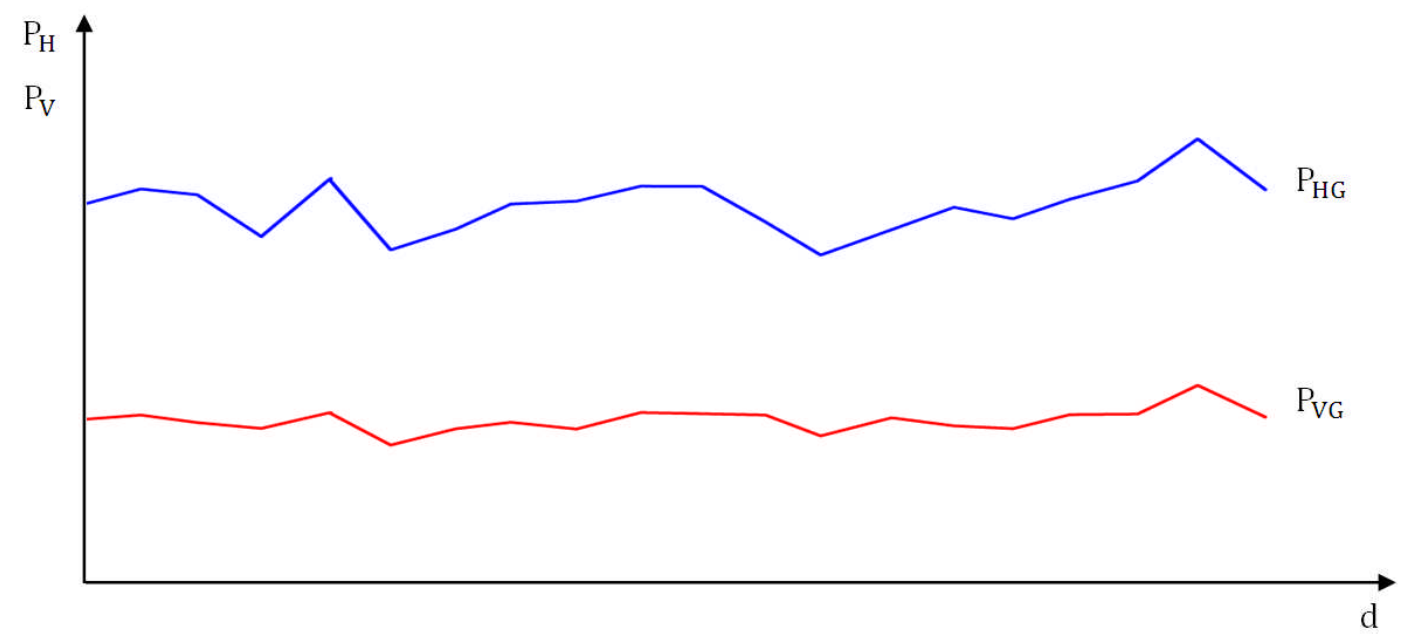

Figure 5: Spatial variability in tillage forces when omitting local tillage forces

538

539

540

541

542

543

544

545

546

547

548 


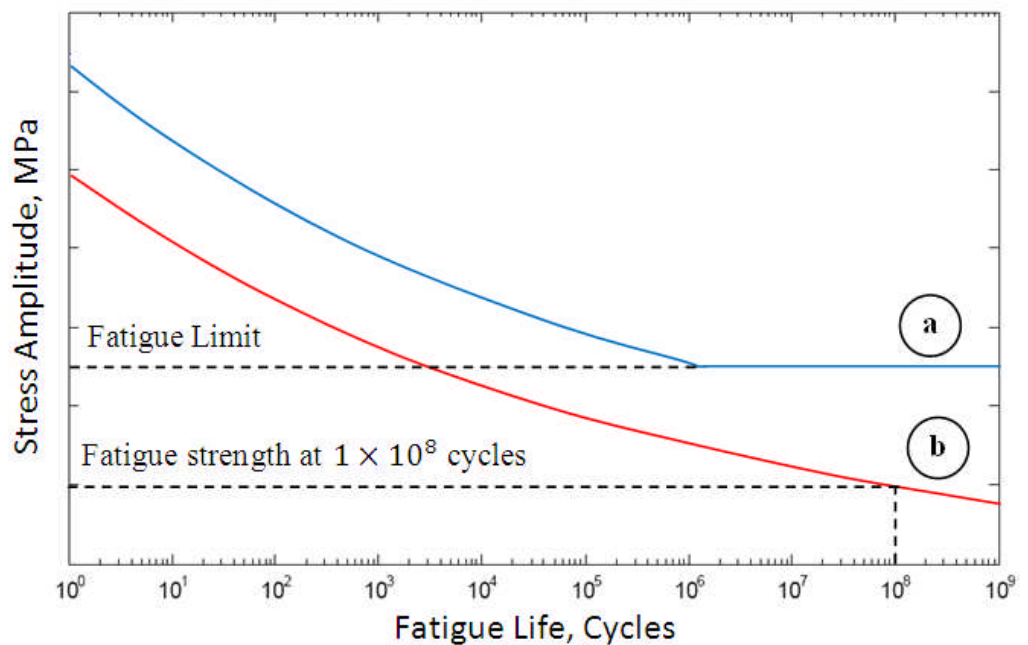

552

Figure 6: Typical S-N curves

553

554

555

556

557

558

559

560

561

562

563 


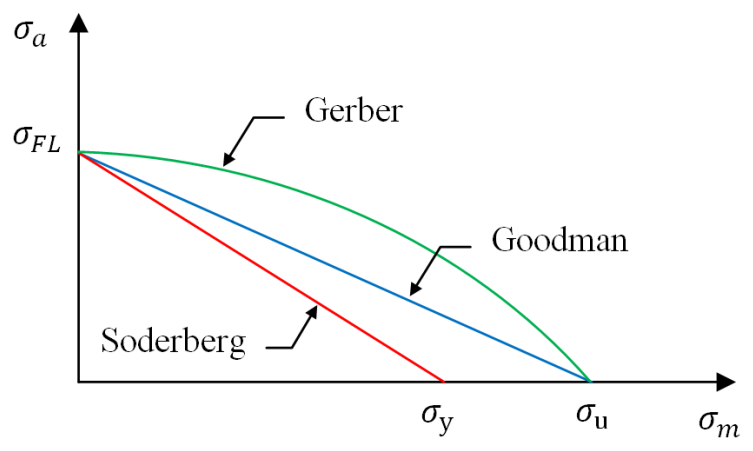

567 (where is the fatigue limit, is the ultimate strength and is the yield strength) 


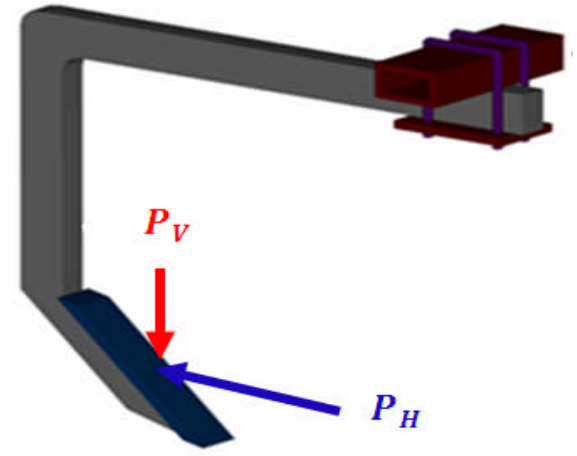

584

586

587

588

589

590

591

592

593

594

595

596

597 


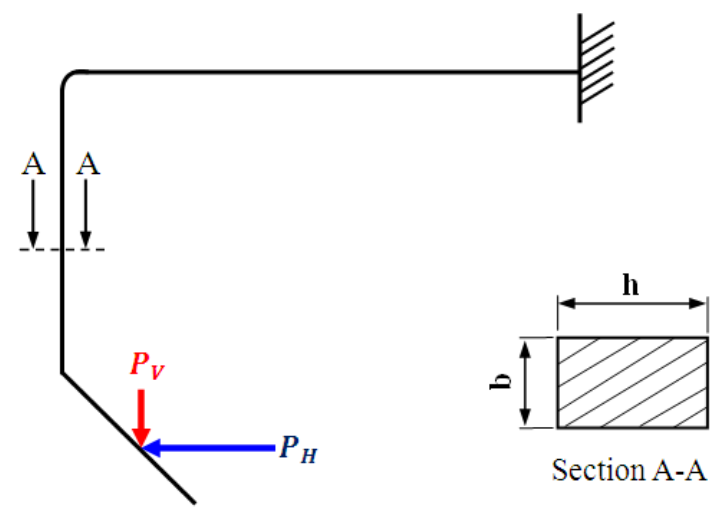

600

Figure 9: A schematic drawing of the chisel plough shank $(h=58$

$=32$

601

602

603

604

605

606

607

608

609

610

611

612

613

614 


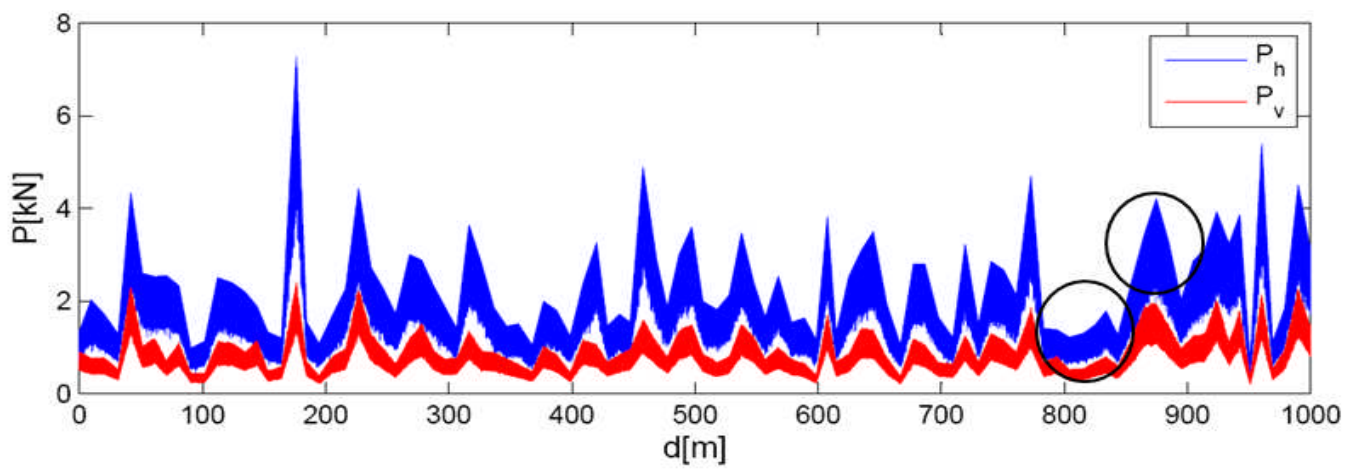

618 Figure 10: The spatial variability of the horizontal and vertical forces across proposed $1000 \mathrm{~m}$ 

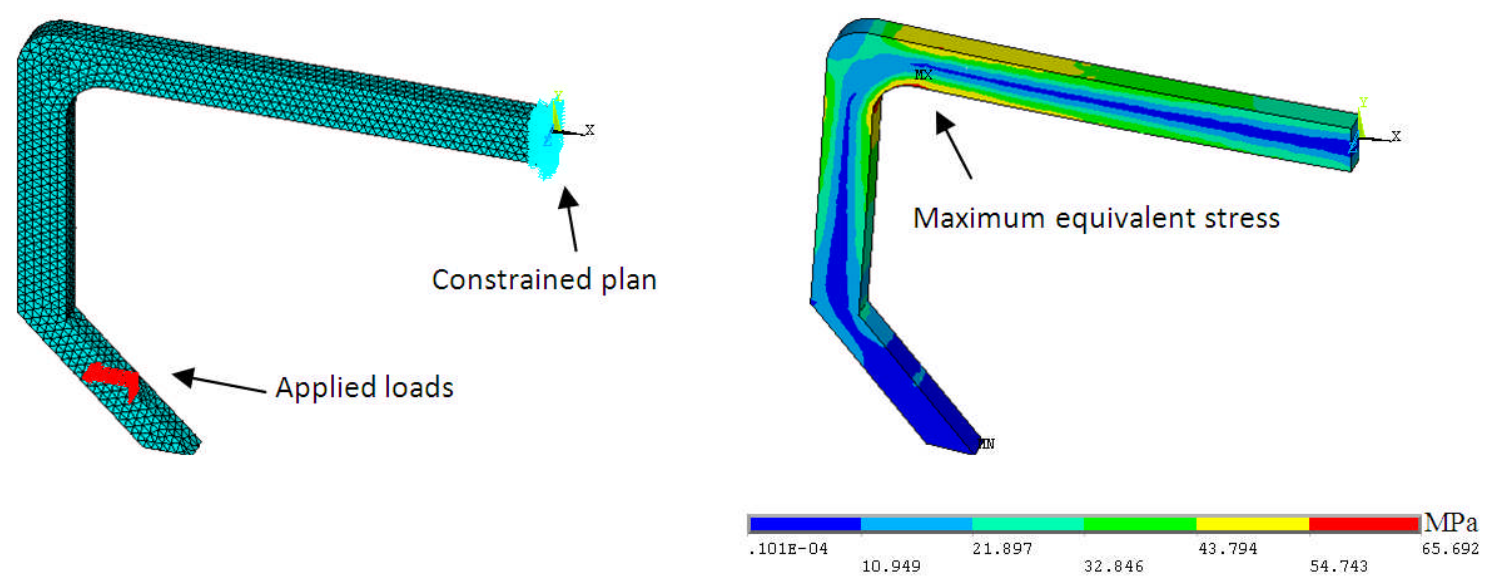

633

a

b

634 Figure 11: a- Meshed model and boundary conditions; b- Point of maximum equivalent stress

635

636

637

638

639

640

641

642

643

644

645

646 


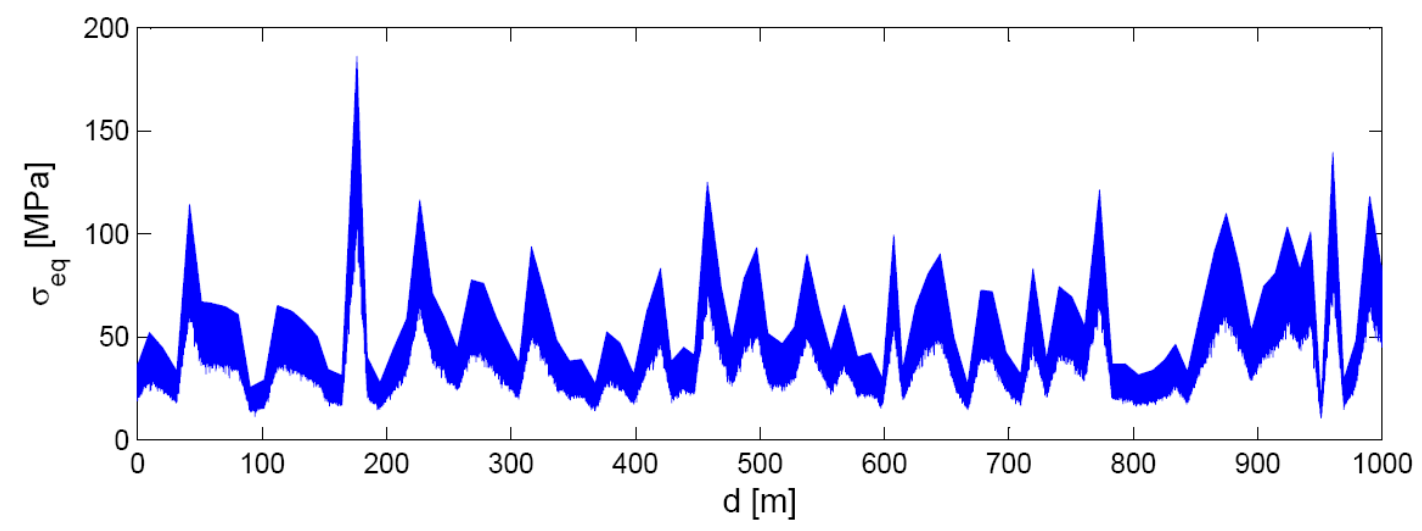

649

Figure 12: Equivalent stress history across proposed $1000 \mathrm{~m}$ distance

650

651

652

653

654

655

656

657

658

659

660

661 

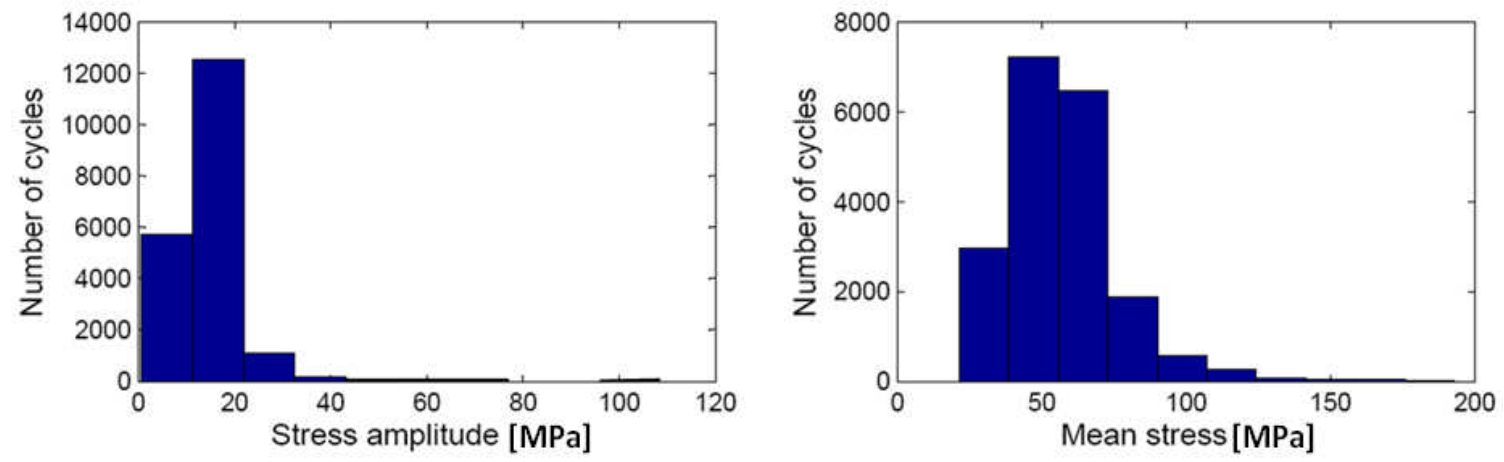

663

Figure 13: Histograms of stress amplitude and mean stress

664

665

666

667

668

669

670

671

672

673

674

675

676

677

678 


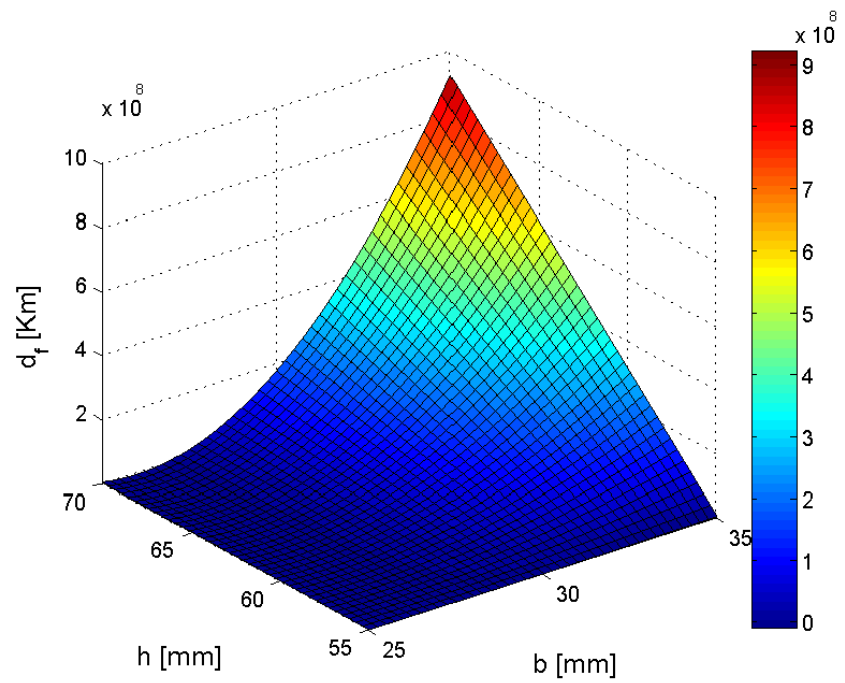

688 


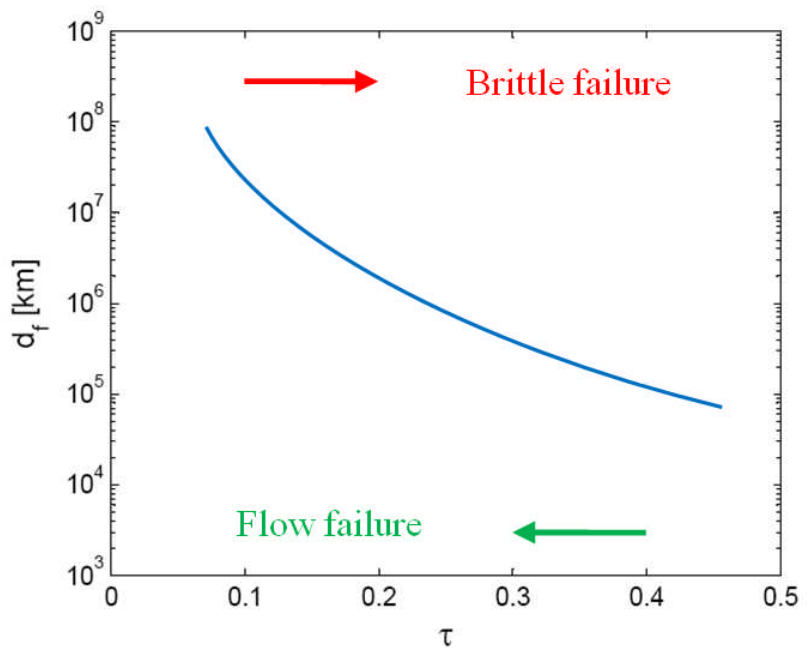

695

696

Figure 15: - relationship

697

698 